\title{
Erratum: Constraints on Hořava gravity \\ from binary black hole observations \\ [Phys. Rev. D 99, 024034 (2019)]
}

Oscar Ramos and Enrico Barausse $[0$

Q (Received 25 August 2021; published 13 September 2021)

DOI: 10.1103/PhysRevD.104.069904

The spin- 0 mode speed in Eq. (17b) has a typo in the denominator. The correct expression is

$$
c_{0}^{2}=\frac{(\lambda+\beta)(2-\alpha)}{\alpha(1-\beta)(2+3 \lambda+\beta)} .
$$

The typo does not propagate, is only present in the paper and not in our calculations, and it does not affect the results in any way. 